\title{
Efecto del diseño de revestimientos sobre el consumo de potencia en molienda ${ }^{(\bullet)}$
}

\author{
W. Valderrama ${ }^{(*)}$ y L. Magne ${ }^{(* *)}$ \\ Resumen Se midió el consumo de potencia en un molino discontinuo de laboratorio de $290 \times 110 \mathrm{~mm}$ cargado \\ con bolas, cilindros o granallas de tamaño uniforme. El ángulo formado por la cara frontal de un \\ levantador y la superficie base de la pieza son el parámetro clave para controlar el consumo de poten- \\ cia. Los mayores valores se obtienen utilizando levantadores con ángulos de $45^{\circ}$ y velocidad de rota- \\ ción del molino de aproximadamente $85 \%$ de la velocidad crítica. Cuando el molino es equipado con \\ levantadores de alto ángulo de ataque, el consumo de potencia decrece y la velocidad de máxima \\ potencia se desplaza a valores más bajos. Para levantadores con ángulos menores de $45^{\circ}$, el consumo \\ de potencia a bajas velocidades se reduce, incrementándose el máximo más allá de la velocidad críti- \\ ca en muchos casos, lo que indica resbalamiento de la carga. La altura del levantador tiene un efecto \\ variable sobre la potencia, dependiendo del ángulo de ataque del levantador y su tamaño relativo \\ frente a los medios de molienda. La mayor altura reduce el consumo máximo de potencia, que se \\ alcanza a una velocidad de giro más baja. Los levantạdores de ángulos menores son menos sensibles \\ a la altura.
}

Palabras clave: Revestimiento de molinos. Consumo de potencia en molienda. Diseño de revestimientos.

\section{Effect of lifter design upon mill power consumption}

\begin{abstract}
Power mill draw was measured in a $290 \times 110 \mathrm{~mm}$ laboratory batch mill, loaded with balls, cylinders or metal grains. Angle between front face of the lifter and its base-surface are the key parameter to control power consumption. A $45^{\circ}$ lifter front angle and about $85 \%$ rotational speed lead to the maximum power draw. As the mill is provided with higher lifter front angles, a decrease in power occurs, and the maximum with respect to the mill velocity is shifted to lower values. For lifter front angle lower than $45^{\circ}$, power consumption at low mill rotational speed is reduced, and the maximum is often moved beyond the critical speed value, which indicates slip of the load. Lifter height has a variable effect upon power draw, depending on the front angle and its relative size compared to the grinding bodies. Generally, an increase in height reduces the maximum power draw, and it is obtained at a lower rotational speed. Low-angle lifters are less sensible to the height. Number of lifter did not show any effect on power draw.
\end{abstract}

Keywords: Mill lifters. Milling power draw. Lifters design.

\section{INTRODUCCIÓN}

La principal función del revestimiento interior de un molino rotatorio es la protección contra el desgaste, constituyendo, además, el medio de transferencia de energía a la carga definiendo en parte las características de moliendabilidad del mineral (1). Así, la magnitud de la energía disponible y su

\footnotetext{
(•) Trabajo recibido el día 18 de julio de 1995.

(*) Dpto. de Ciencia de Materiales. Universidad Técnica Federico Santa María. Avda. España 1680. Casilla 110-V. Valparaíso (Chile).

(**) Dpto. de Ingeniería Metalúrgica. Universidad de Santiago de Chile. Avda. B. O'Higgins 3363. Casilla 10233. Santiago (Chile).
}

distribución en el seno del molino determinan la capacidad de molienda mediante el movimiento de la carga (2). El diseño del perfil del revestimiento modifica las trayectorias seguidas por los componentes de la carga, debiendo influir entonces sobre el consumo de potencia y la capacidad de molienda.

La bibliografía consigna numerosos trabajos sobre este tema. Sobre la relación entre energía disponible y la velocidad de molienda, Rose y Sullivan (3) y luego Leluschko (4) mostraron que la velocidad de creación de nueva superficie y el consumo de potencia se encuentran fuertemente correlacionados. La presencia de levantadores en el revestimiento modifica tanto la pendiente de la curva de potencia frente a la velocidad, como la velocidad a la cual se obtiene la potencia máxima. En molinos de 
superficie interna lisa, esta pendiente es menor que en molinos provistos de levantadores (5). La curva de potencia en molinos de superficie interna lisa presenta un máximo más allá de la velocidad crítica, seguido generalmente de una caída abrupta (6).

El efecto de la altura de levantadores sobre la distribución de energía dentro del molino se ha estudiado experimentalmente por Rolf y Simonis (7), encontrando una buena correlación entre el par (torque) máximo aplicado al molino y la máxima frecuencia de impactos de bola de alta energía. Este máximo se desplaza hacia velocidades menores al aumentar la altura de levantadores. Meaders y Macpherson (8) hallaron una dependencia del consumo de potencia y la capacidad del molino en función de la razón de altura y separación de levantadores. Ellos dieron origen al valor de diseño hoy ampliamente aceptado de 4:1 para este parámetro.

Las anormalidades en el consumo de potencia asociadas a molinos de superficie interna lisa se atribuyen normalmente al resbalamiento de la carga. Manz (9) observó resbalamiento cuando el nivel de llenado de bolas es inferior al $40 \%$ y se aumenta la velocidad del molino. La presencia de mineral modifica sólo levemente esta situación. Vermeulen y Howat (10) midieron el resbalamiento en un molino de barras de laboratorio con y sin levantadores. Sus resultados indican que el resbalamiento está siempre presente. Los levantadores sólo evitan el resbalamiento de las capas externas de la carga respecto de la carcasa.

$\mathrm{Al}$ examinar la influencia de los levantadores, la mayor parte de los autores han comparado molinos sin levantadores con molinos provistos de un número variable de levantadores rectangulares de una altura determinada. En algunos casos se han usado otras geometrías: Austin et al. (11) describen ensayos realizados con levantadores semicirculares. Fuerstenau y Abouzeid (12) hicieron mediciones de cinética de molienda usando levantadores de varias alturas y un ángulo constante de $45^{\circ}$ entre la carcasa y la cara frontal del levantador (ángulo de ataque). Sinel'nikova y Puchkova (13) describieron ensayos de perfiles a escala industrial (ondulado, levantador y placa, espiral angular) encontrando curvas de potencia con pendientes similares para los tres diseños y un mayor consumo de potencia a una velocidad más alta que los otros diseños para la forma ondulada. La velocidad de molienda de material fino fue también mayor para el perfil ondulado.

No obstante, la influencia de la forma del revestimiento sobre el consumo de potencia no ha sido estudiada en forma sistemática. El propósito de este trabajo es presentar nueva información experimental del efecto del perfil del revestimiento sobre el consumo de potencia y discutir en forma preliminar los mecanismos que afectan este fenómeno.

\section{TRABAJO EXPERIMENTAL}

Las condiciones en que se realizaron los diversos ensayos aparecen resumidas en la tabla I. Para los ensayos se utilizó un molino provisto de levantadores de cara plana. El ángulo de ataque se mide entre la base de la pieza y la cara frontal, como indica la figura 1. La altura de los levantadores se seleccionó de modo que correspondiera a un medio, uno y dos diámetros de las bolas utilizadas. El molino permite instalar un número variable de levantadores. La carga consistió en bolas de acero de diámetro uniforme, granalla redondeada para minimizar efectos de impacto y cilindros.

Las determinaciones de potencia se hicieron con ayuda de un sensor de par torsión y corregidas para descontar pérdidas por fricción. Cada resultado representa el promedio de 400 medidas que se transferían directamente a un ordenador. Ensayos de control mostraron desviaciones siempre inferiores al $3 \%$.

\section{RESULTADOS}

El efecto de los levantadores bajo diferentes condiciones de operación en el consumo de potencia se presenta gráficamente en las figuras 2 a 6 . La figura 2 muestra el efecto del ángulo de ataque sobre el consumo de potencia. En la figura 2a) se han representado en forma de gráficos los datos obtenidos con un $40 \%$ de nivel de llenado de bolas

TABLA I.- Condiciones experimentales

TABLE I.- Experimental conditions

\begin{tabular}{|c|c|}
\hline Molino & $\begin{array}{l}290 \mathrm{~mm} \text { de diámetro interior } \\
110 \mathrm{~mm} \text { de largo interior }\end{array}$ \\
\hline $\begin{array}{l}\text { Nivel de } \\
\text { llenado }\end{array}$ & $\begin{array}{l}30 \% \text { del volumen interior } \\
40 \% \text { del volumen interior }\end{array}$ \\
\hline Carga & $\begin{array}{l}\text { Medios de molienda de tamaño } \\
\text { uniforme: } \\
\text { - bolas de } 6 \mathrm{~mm} \\
\text { - bolas de } 9,5 \mathrm{~mm} \\
\text { - granalla redondeada de } 1,5 \mathrm{~mm} \\
\text { - cilindros de } 10 \times 10 \mathrm{~mm}\end{array}$ \\
\hline $\begin{array}{l}\text { Ángulo de } \\
\text { ataque de } \\
\text { levantadores }\end{array}$ & $30^{\circ} ; 45^{\circ} ; 60^{\circ} ; 90^{\circ} ; 120^{\circ} ; 135^{\circ}$ \\
\hline $\begin{array}{l}\text { Altura de } \\
\text { levantadores }\end{array}$ & $\begin{array}{l}6,10,20 \mathrm{~mm}(1 / 2,1 \text { y } 2 \text { veces el } \\
\text { diámetro de bolas de } 9,5 \mathrm{~mm})\end{array}$ \\
\hline $\begin{array}{l}\text { Núm. de } \\
\text { levantadores }\end{array}$ & 12,16 y 24 \\
\hline
\end{tabular}




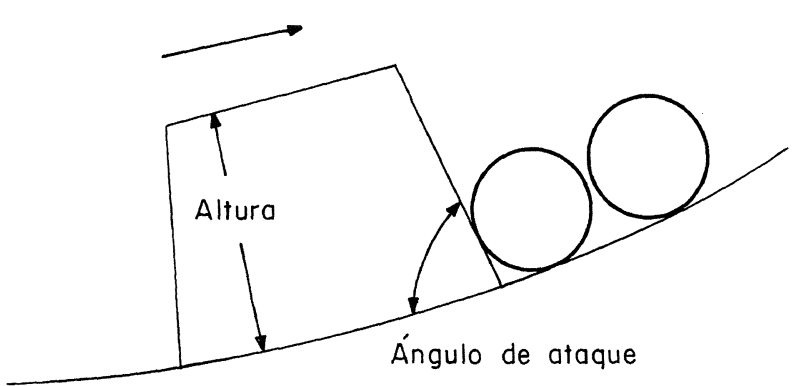

FIG. 1.- Definición de magnitudes características de un levantador.

FIG. 1.-Definition of characteristic magnitudes of lifter.

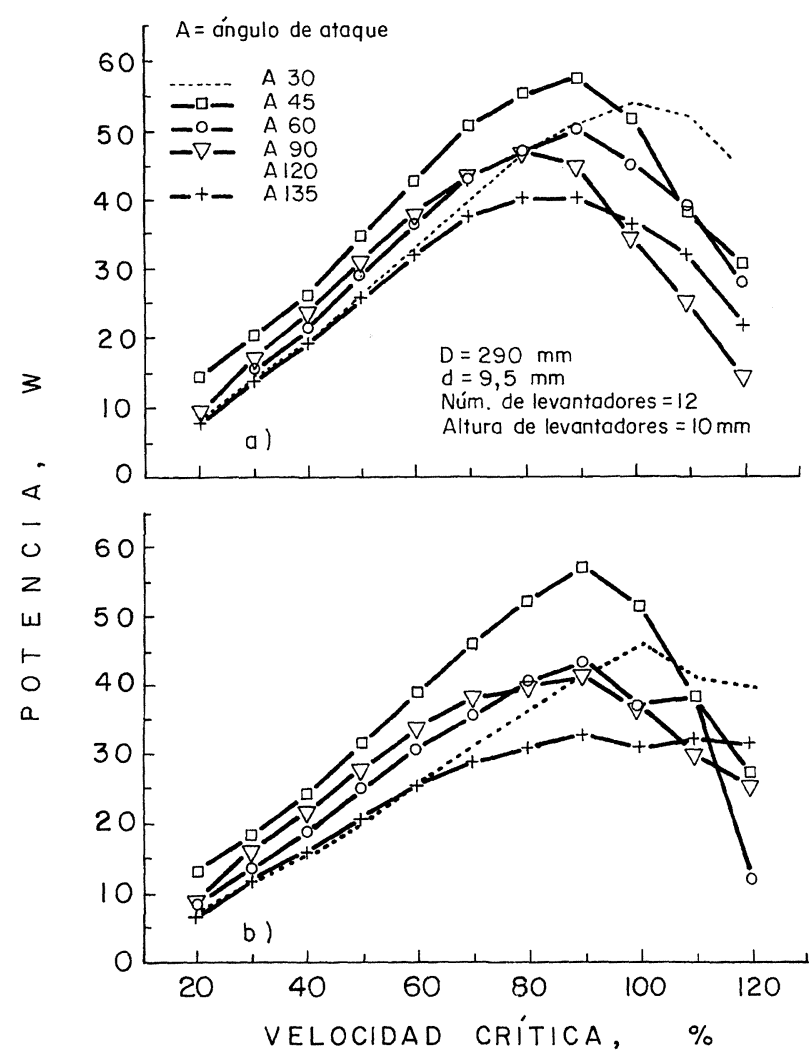

FIG. 2.- Curvas de consumo de potencia en función de la velocidad de rotación para diferentes ángulos de ataque. Nivel de llenado de bolas. a) $40 \%$. b) $30 \%$..

FIG. 2.- Power consumption versus mill speed curves for different lifter front angles. Volume mill filling.

a) $40 \%$.b) $30 \%$.

de $9,5 \mathrm{~mm}$, con levantadores de $10 \mathrm{~mm}$ de altura, equivalente a un diámetro de bola. El mayor consumo de potencia se obtiene con levantadores de ángulo de ataque de $45^{\circ}$. Al aumentar el ángulo de ataque $\left(60^{\circ}\right.$ o más), se observa una disminución creciente del consumo de potencia. También se observa que el máximo de la curva se desplaza hacia valores más bajos a medida que aumenta el ángulo de ataque $\left(45^{\circ}, 60^{\circ}, 90^{\circ}\right)$. Una conducta diferente exhibe el levantador de ángulo de $30^{\circ}$ al ser la curva más baja de todo el conjunto, evidenciando una menor transferencia de energía a la carga. A la vez, el máximo se encuentra cerca del $100 \%$ de la velocidad crítica. Ambos hechos indican la presencia de resbalamiento en el seno de la carga. La figura $2 \mathrm{~b}$ ) muestra los datos correspondientes a un $30 \%$ de llenado del molino. Aunque los valores de potencia consumida son menores que los anteriores, el orden relativo es el mismo: máximo consumo para ángulo de $45^{\circ}$, consumos decrecientes para ángulos de ataque mayores y una curva con señales de resbalamiento para el ángulo de $30^{\circ}$.

La altura del levantador presenta un efecto que depende del ángulo de ataque, según muestra la figura 3. Para ángulos bajos (Fig. 3a), $30^{\circ}$ y $45^{\circ}$ ), la altura del levantador prácticamente no tiene efecto. En cambio, cuando el ángulo de ataque es de $90^{\circ}$ (Fig. 3b), la mayor altura reduce el consumo de

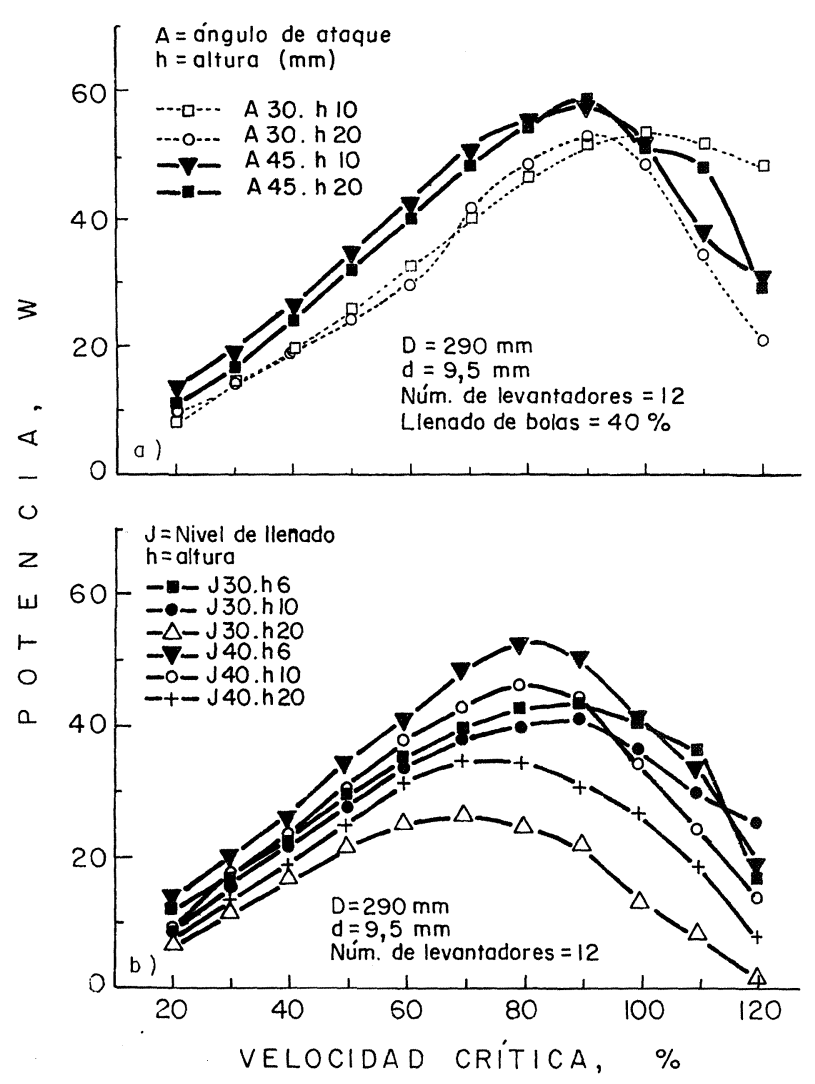

Fig. 3.- Curvas de consumo de potencia en función de la velocidad de rotación para diferentes alturas de levantador. Ángulos de ataque:

$$
\text { a) } 45^{\circ} \mathrm{C} \text { y } 30{ }^{\circ} \mathrm{C} \text {. b) } 90{ }^{\circ} \mathrm{C} \text {. }
$$

FIG. 3.- Power consumption versus mill speed curves for different lifter height. Lifter front angles. a) $45^{\circ} \mathrm{C}$ and $30^{\circ} \mathrm{C}$. b) $90^{\circ} \mathrm{C}$. 
potencia. El efecto es más marcado cuando la altura aumenta de uno a dos diámetros de bola, respecto del cambio de un medio a un diámetro.

El número de levantadores (Fig. 4) no mostró un efecto relevante. Las diferencias que se aprecian más allá del máximo de la curva de potencia son de poco interés práctico, y corresponden a una zona inestable para las mediciones.

El tamaño de los medios de molienda (Fig. 5) tampoco muestra un efecto relevante. Los valores medidos con granalla debieron ser corregidos debido a la menor densidad de este material.

La forma de los medios de molienda (Fig. 6) presenta un efecto dependiente del ángulo de ataque. Para ángulo de ataque de $45^{\circ}$, bolas y cilindros presentan un mismo consumo de potencia a baja velocidad. A $80 \%$ de la velocidad crítica, el consumo de potencia alcanza un máximo para los cilindros, mientras que con bolas, este fenómeno ocurre a un $90 \%$ de la velocidad crítica. Cuando el ángulo de ataque es de $90^{\circ}$, los cilindros muestran también máximos de consumo de potencia a velocidades menores que las bolas cuando las demás condiciones se mantienen constantes. A baja velocidad, los cilindros consumen un poco más de potencia que las bolas. Este efecto es muy notorio para la altura de $20 \mathrm{~mm}$ (dos diámetros de bola) y $30 \%$ de llenado, lo que sugiere la presencia de resbalamiento. En la figura 6 , los valores de consumo de potencia de cilindros se han corregido para considerar una densidad aparente levemente mayor que las bolas, aunque tal efecto resultó finalmente irrelevante $(2,4 \%)$.

\section{DISCUSIÓN}

Los resultados anteriores indican que el revestimiento permite modificar en forma relevante el

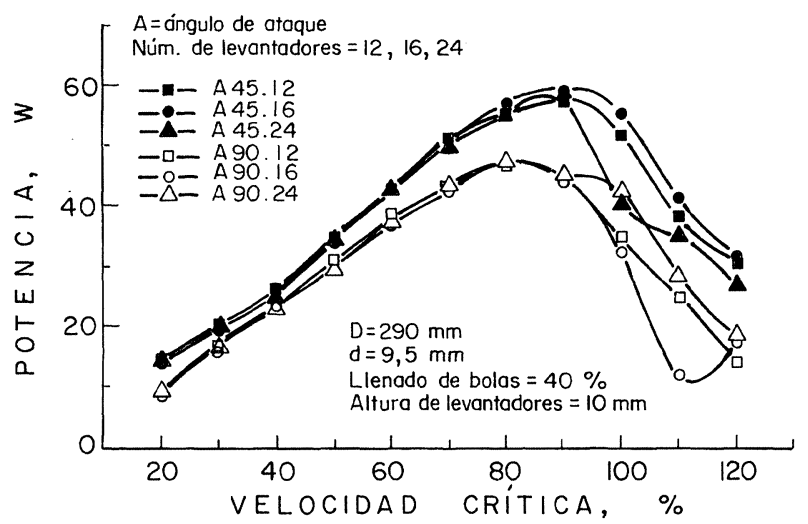

FIG. 4.- Curvas de consumo de potencia en función de la velocidad de rotación para diferentes distancias entre levantadores.

FIG. 4.- Power consumption versus mill speed curves for different distances between lifters.

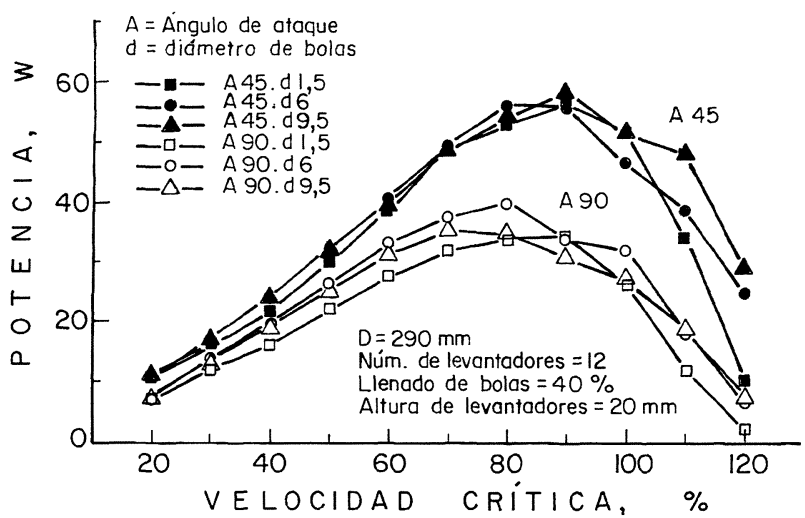

FIG. 5.- Curvas de consumo de potencia en función de la velocidad de rotación para diferentes tamaños medios de molienda y ángulo de ataque del levantador.

FIG. 5.- Power consumption versus mill speed curves for different grinding media size and lifter front angles.

consumo de potencia del molino. Una tratamiento teórico cuantitativo de estos resultados está fuera del alcance de este trabajo; sin embargo, es posible proponer algunas vías de explicación tomando como base las trayectorias de la carga, según han sido observadas directamente en el molino y calculadas por M. S. Powell (14).

Powell ha descrito matemáticamente la interacción de un levantador de cara plana con una bola. Su enfoque establece cuatro etapas (Fig. 7a):

- Ascenso de la bola pegada a la superficie interior del molino debido a la acción simultánea de las fuerzas de gravedad, centrífuga y de roce. $\mathrm{Al}$ llegar a una determinada altura, la fuerza neta se anula, iniciando la segunda etapa.

- Rodado de la bola sobre la superficie del levantador, sostenida por la fuerza de roce. A medida que continúa el ascenso, aumenta la fuerza resultante que actúa sobre la bola, hasta el punto en que supera la fuerza de roce y comienza la tercera etapa,

- Deslizamiento de la bola sobre la superficie, hasta alcanzar el borde del levantador o hasta que se anule la fuerza de contacto por alcanzar la vertical la superficie del levantador. En cualquiera de ambos casos, se da comienzo a la cuarta etapa,

- Caída libre de la bola.

Cuando el ángulo de ataque es pequeño, ocurre que las trayectorias predichas por este modelo se ubican por debajo de la superficie de la carga. En ese caso, las predicciones no son válidas y las bolas se mueven siguiendo trayectorias determinadas por la interacción con las vecinas, generalmente del tipo de cascada. Las figuras 7b), c) y d) muestran el efecto de la velocidad, del ángulo de ataque y de la 

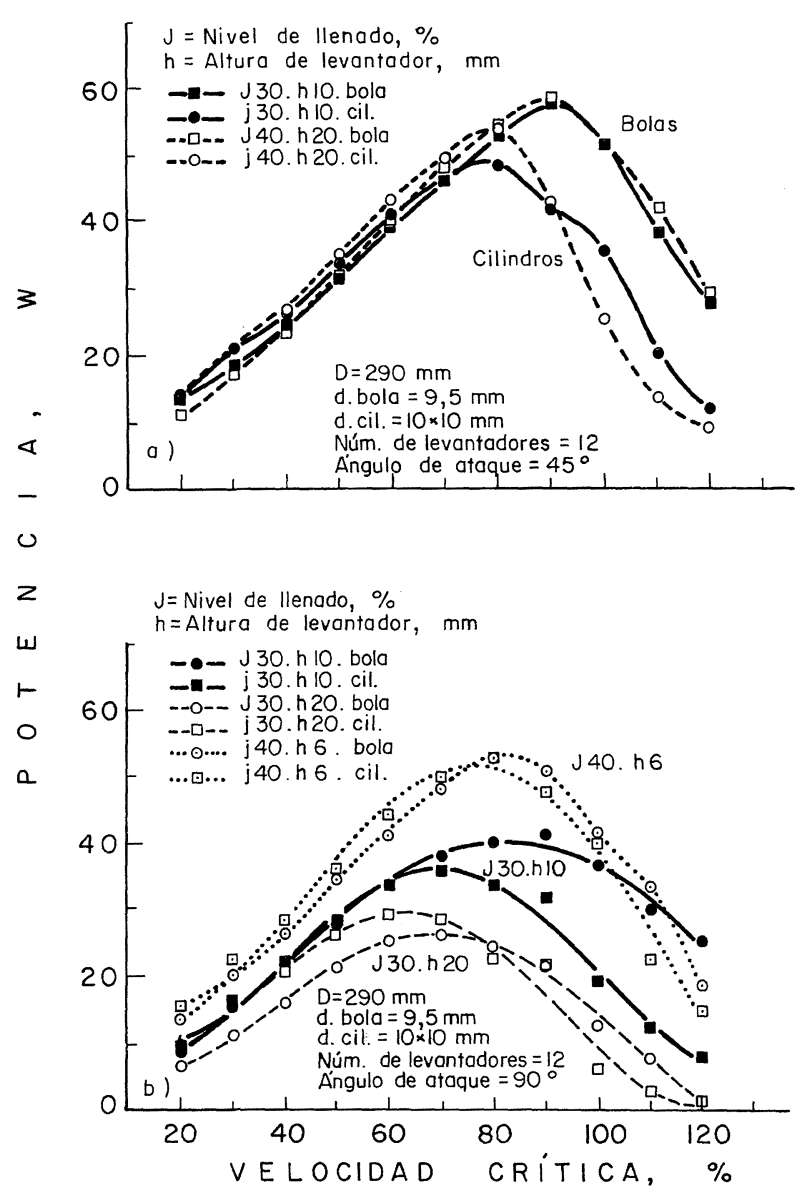

Fig. 6.- Curvas de consumo de potencia en función de la velocidad de rotación para diferentes formas del medio de molienda y ángulo de ataque del levantador.

a) $45^{\circ} \mathrm{C}$. b) $90{ }^{\circ} \mathrm{C}$.

FIG. 6.- Power consumption versus mill speed curves for different grinding media shape and lifter front angles.

a) $45^{\circ} \mathrm{C}$. b) $90^{\circ} \mathrm{C}$.

altura sobre las trayectorias de caída, según Powell. La velocidad y el ángulo de ataque muestran un efecto considerable, mientras que la altura tiene un efecto que se satura rápidamente.

La figura 8 muestra fotografías de las trayectorias de los elementos de la carga para dos ángulos de ataque típicos $\left(45^{\circ}\right.$ y $\left.90^{\circ}\right)$. Allí se puede observar claramente que el mayor ángulo de ataque genera un mayor levantamiento de la carga contenida en la capa exterior para una velocidad de giro dada. Como consecuencia de ello, se requieren velocidades más bajas para que comience el impacto directo de la carga sobre la superficie interior del molino.

El alcance de un máximo de la curva de potencia está asociado a la transferencia de cantidad de movinmiento al eje del molino durante el impacto, lo cual reduce la demanda neta de energía externa. Este factor se hace creciente con la velocidad, hasta el punto en que la carga se centrifuga y la energía que se acumula en la etapa de ascenso se restituye al eje durante el descenso, menos las pérdidas.

A partir de esta observación resulta fácil explicar el efecto del ángulo de ataque que muestra la figura 2. La reducción del ángulo de ataque permite retardar hasta mayores velocidades de giro el comienzo de la retrotransferencia de cantidad de movimiento al molino, permitiendo a la carga alcanzar niveles más altos de potencia (Figs. 7c) y b)). Por otra parte, por esta misma razón, la velocidad a la cual se genera el máximo aumenta al reducir el ángulo de ataque. Esta afirmación no es válida para un ángulo de ataque de $30^{\circ}$ o menos, debido a la presencia probable de resbalamiento, que se hace notorio por el menor consumo de energía en la zona ascendente de la curva de potencia en relación a ángulos de ataque mayores. Por otra parte, también el máximo de la curva aparece a velocidades notoriamente superiores a la crítica. Ambos fenómenos indican que la carga no fue levantada hasta una altura adecuada en el momento en que la fuerza de roce asociada a la fuerza centrífuga alcanzó el valor apropiado, a velocidades comparativamente altas.

El efecto de la altura del levantador varía con el ángulo de ataque. Para un ángulo de $45^{\circ}$, la mayor altura tiene un efecto mínimo, asociado probablemente al menor grosor de la capa no afectada por el levantador, lo que favorece el deslizamiento. La causa de esto radicaría en que a altas velocidades las bolas se desprenden antes de alcanzar el borde del levantador, haciendo, por ende, su altura irrelevante. A velocidades menores, el despegue de la carcasa ocurre bajo la superficie, siguiendo la bola una trayectoria determinada por las vecinas, independientemente del levantador. Con ángulos de ataque menores de $45^{\circ}$ ocurre lo mismo. Para ángulos de ataque mayores, el proceso se traslada por encima de la superficie de la carga, sin que se despegue la bola antes de alcanzar el borde extremo; por ello, el efecto de la altura es mucho más perceptible. Sin embargo, cuantitativamente el efecto es más notorio al pasar de $1 / 2$ a 1 diámetro de bola. La figura $7 d$ ) muestra el escaso efecto de pasar de una altura de uno a dos diámetros de bola. El efecto debe tener entonces una componente propia del molino de laboratorio: al disminuir el diámetro y el nivel de llenado efectivos del molino se favorece el resbalamiento.

El número de levantadores no tiene efecto en la medida en que no cambian las trayectorias de las bolas al caer y el tamaño de medios de molienda tiene un efecto muy limitado. Geométricamente, al aumentar el tamaño de los medios se reduce la 


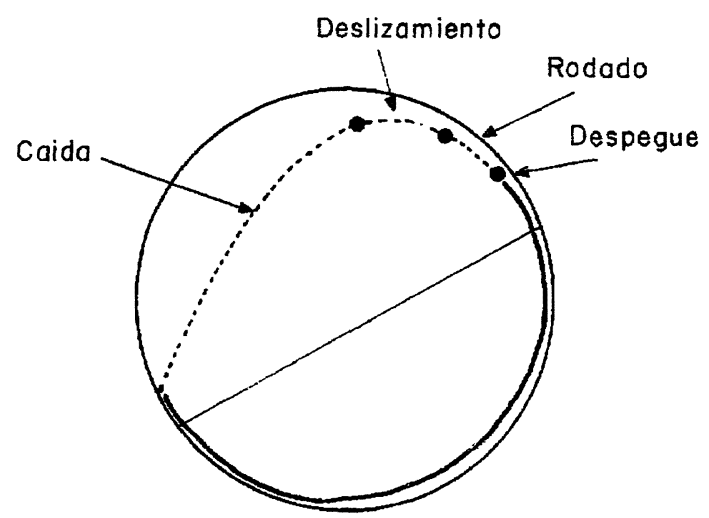

a)
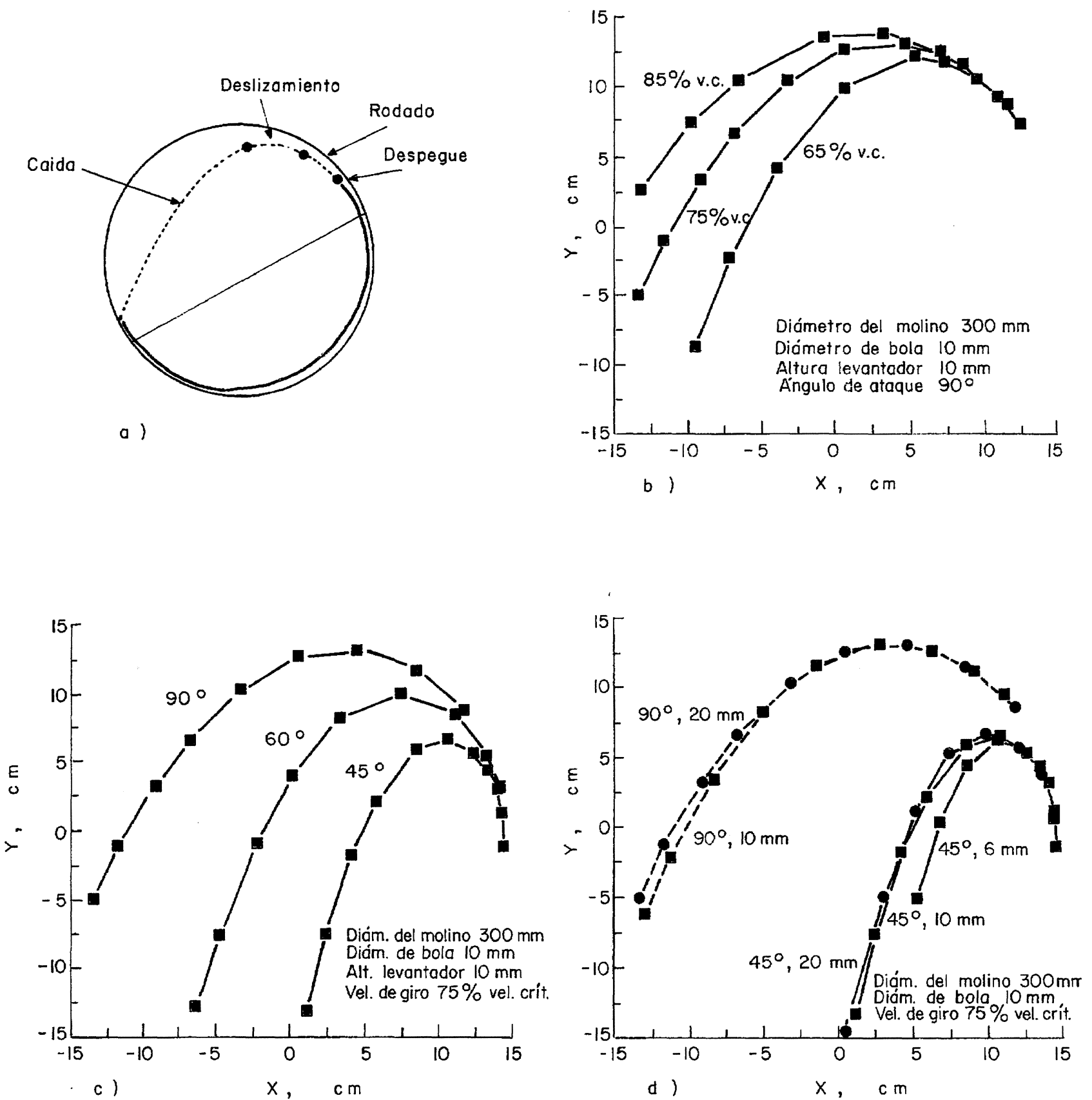

FIG. 7.-Efecto de las condiciones relevantes de diseño y operación en las trayectorias de caída de una bola.

a) Intercara entre bola y levantador.

b) Influencia de la velocidad.

c) Efecto del ángulo de ataque.

d) Influencia de la altura de los levantadores.

FIG. 7.- Effect of relevant design and operational conditions in the ball falling path.

a) Interface between ball and lifter.

b) Effect of speed.

c) Effect of lifter front angle.

d) Effect of lifter height.

altura relativa del levantador, actuando éste como si fuera más pequeño, aumentando la velocidad del máximo de potencia cuando el ángulo de ataque es mayor. Para ángulos de ataque menores $\left(45^{\circ}\right)$, el tamaño de medios de molienda tiene una influencia más limitada. La granalla mostró en ese caso un 

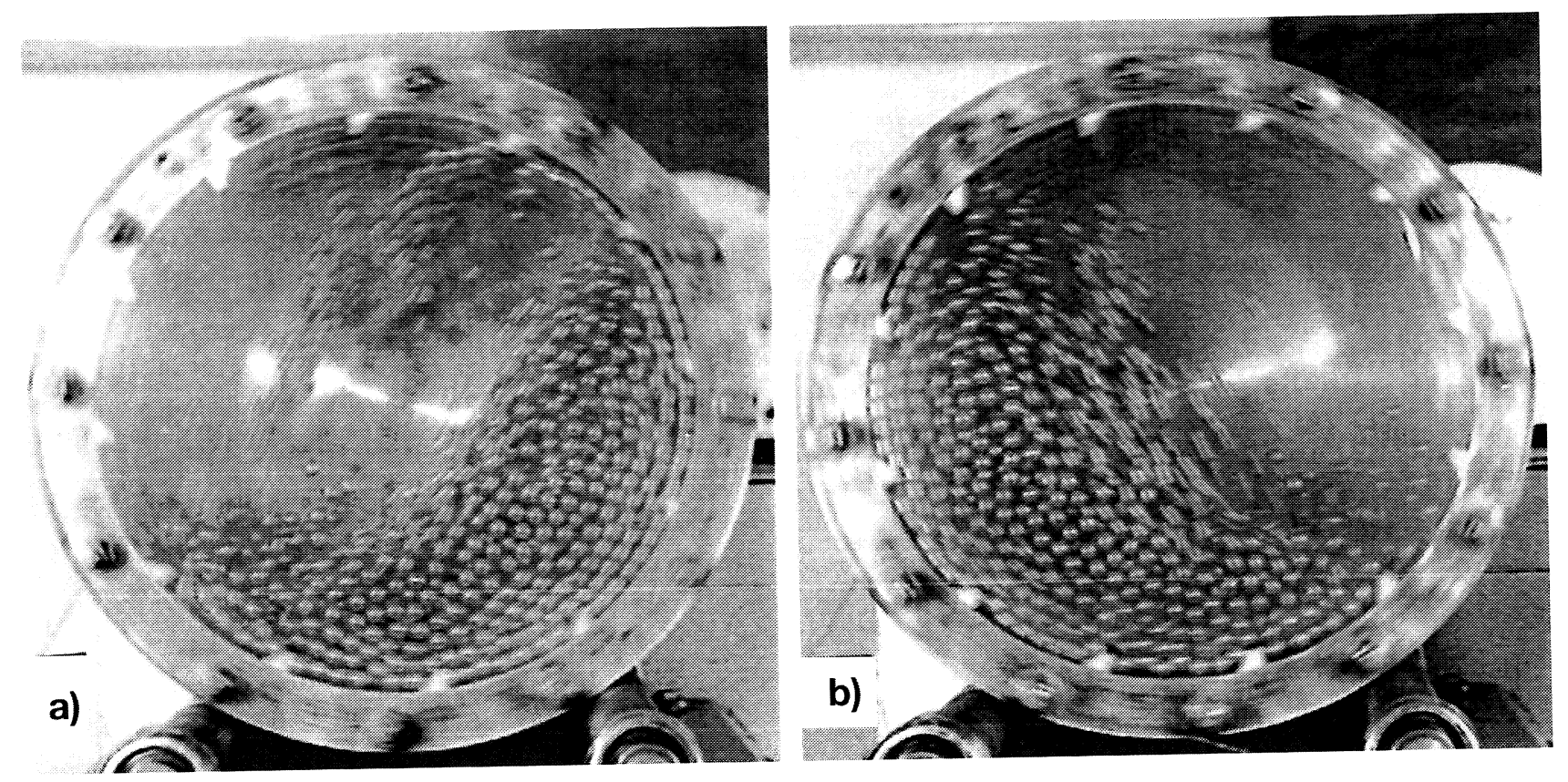

FIG. 8. - Movimiento de la carga para el molino con un $30 \%$ de llenado.

a) ángulo de ataque de $90^{\circ}$ y $70 \%$ de la velócidad crítica.

b) ángulo de ataque de $45^{\circ}$ y $80 \%$ de la velocidad crítica.

FIG. 8.-Mill load motion for $30 \%$ filling.

a) $90^{\circ}$ lifter front angle and $70 \%$ of the critical speed.

b) $45^{\circ}$ lifter front angle and $80 \%$ of the critical speed.

consumo de potencia equivalente mayor al tomar en cuenta su menor densidad, desviándose del comportamiento general.

La forma del medio de molienda se utiliza para obtener información acerca del deslizamiento en el seno de la carga. Este comportamiento se refleja en el ángulo de talud de la carga. Los valores medidos para cargas de bolas y cilindros de tamaño uniforme fueron 30 y $42^{\circ}$, respectivamente; es decir, los cilindros muestran una mayor resistencia al desmoronamiento y alcanzan mayores alturas a iguales condiciones que las bolas. Por lo mismo, los máximos de potencia deben situarse a velocidades menores, lo que efectivamente se verifica en todos los casos incluidos en la figura 6.

\section{CONCLUSIONES}

- El revestimiento influye en forma relevante en el consumo de potencia de un molino, en magnitud que puede alcanzar un $100 \%$ de variación.

- La variable más influyente es el ángulo de ataque, que muestra máximos consumos para valores de $45^{\circ}$ de este parámetro.

- La altura del levantador tiene importancia cuando el ángulo de ataque es alto (cercano a $90^{\circ}$ ), y la altura del levantador supera un diámetro de bola.
- La separación entre levantadores no mostró ningún efecto importante.

- El tamaño y la forma de medios de molienda muestran una influencia que es modificada por la forma del revestimiento.

- Los efectos antes señalados se relacionan con la trayectoria de bolas en contacto directo con el levantador, según el modelo desarrollado por Powell.

\section{Agradecimiento}

Este trabajo ha sido financiado por FONDECYT, a través de los proyectos 89-260 y 92-582, y la Universidad Técnica Federico Santa María, a través del proyecto 902106 . Los autores agradecen a la Universidad de Santiago de Chile la oportunidad brindada para desarrollar este trabajo y, en especial, a los Profesores Leonard Austin y Fernando Concha por su ayuda.

\section{REFERENCIAS}

(1) Magne, L., Valderrama, W., Quintero, M., y MenaCHO, J. Mill Lifter Design and Grinding Kinetics, VI Meeting of the Southern Hemisfere Mineral Technology, y III Latin-American Congress in Froth Flotation, Concepción (Chile), 20-23 nov. 1994: 69-79. 
(2) Valderrama, W., Grandela, P. y Magne, L. Liner Shape and Power Consumption in Rotary Mills, XIX International Mineral Processing Congress. San Francisco CA, (EE.UU.), 22-27 oct. 1995.

(3) Rose, H.E. y Sullivan, R.M.E. A Treatise on the Internal Mechanics of Ball, Tube and Rod Mills, Constable, Londres 1958: 183-186.

(4) LeluschKo, J. Untersuchungen zum Einfluß der Flüssigkeit bei der Naßmahlung in Kugelmühlen, Dissertation, T.U. Munich (R.F.A.) 1984: 78-79.

(5) Rose, H.E. y Sullivan, R.M.E., A Treatise on the Internal Mechanics of Ball, Tube and Rod Mills, Constable, Londres 1958: 73-74.

(6) LeluschKo, J. Untersuchungen zum Einfluß der Flüssigkeit bei der Naßmahlung in Kugelmühlen, Dissertation, T.U. Munich (R.F.A.) 1958: 84-87.

(7) Rolf, L. y Simonis, W. Energy Distribution in Ball Mills, 7th European Symposium Comminution, Ljubljana (Yugoslavia) 12-14 junio 1990.
(8) MEAdERS, R.C. y MacPherson, A.R. Technical Design of Autogenous Mills, Min. Eng., Nueva York, 16 sep. 1964: 81-83.

(9) MANZ, R. DECHEMA Monogr, 69, 1972: 719-749.

(10) Vermeulen L.A. y Howat, D.D. Int. J. Miner. Process., 24, 1988: 143-159.

(11) Austin, L.G., Klimpel, R.R. y Luckie, P.T. Process Engineering of Size Reduction: Ball Milling, Soc. Min. Eng. AIME, Nueva York, NY, 1984: 238-239.

(12) Fuerstenau, D.W. y Abouzeid, A.-Z.M. Int. J. Miner. Process., 15, 1985: 183-192.

(13) Sinel'nikova, L.N. y Puchkova, M.V., Effect of liner shape upon ball mill efficiency. Sov. J. Non-Ferrous Met., 25 (9), 1984: 101-102.

(14) Powell, M.S. Int. J. Miner. Process., 31, 1991: 163-193. 